\title{
Effects of Garlic Oil on the Migration of Neutrophil-Like Cell Studied by Using a Chemotactic Gradient Labchip
}

\author{
Po-Chen Shih, ${ }^{1}$ Chia-Hao Kuo, ${ }^{2}$ Jenh-Yih Juang, ${ }^{1}$ Cheng-Hsien Liu, ${ }^{3}$ \\ Long Hsu, ${ }^{1}$ and Cheng-Tzu Liu' ${ }^{2}$ \\ ${ }^{1}$ Department of Electrophysics, National Chiao Tung University, Hsinchu 300, Taiwan \\ ${ }^{2}$ School of Nutrition, Chung Shan Medical University, Taichung 402, Taiwan \\ ${ }^{3}$ Department of Power Mechanical Engineering, National Tsing Hua University, Hsinchu 300, Taiwan
}

Correspondence should be addressed to Long Hsu, long@cc.nctu.edu.tw

Received 14 September 2009; Revised 23 December 2009; Accepted 14 February 2010

Academic Editor: P. Bryant Chase

Copyright (c) 2010 Po-Chen Shih et al. This is an open access article distributed under the Creative Commons Attribution License, which permits unrestricted use, distribution, and reproduction in any medium, provided the original work is properly cited.

\begin{abstract}
We have designed and fabricated a novel chemotactic gradient Labchip for studying cell migration quantitatively. Owing to the great potential of garlic and its preparations in developing antiinflammatory drugs, the aim of the present study is to investigate the effect of garlic oil on the locomotion of a neutrophil-like cell by measuring the dynamic features of cell migration including migration direction, average migration speed, chemotactic index (CI), and motility index (MI) with the newly designed Labchip. We found that garlic oil treatment lowered the values of CI and MI and reduced the average speed of cell migration from 13 to $8 \mu \mathrm{m} / \mathrm{min}$. The results indicate that garlic oil is a potential inhibitor for neutrophil-like cell migration and chemotactic responsiveness. By comparing with the effects of nocodazole and cytochalasin B, we also suggest that the antiinflammatory activity exhibited by garlic oil was mainly through inhibiting the assembly-disassembly processes of the cytoskeleton.
\end{abstract}

\section{Introduction}

Many fundamental biological mechanisms such as cell migration and chemotaxis are induced by the concentration gradients of either diffusible chemicals or molecules. For example, when local inflammation occurs, the injured tissue will release a fair amount of chemoattractant, a diffusible chemical, to attract neutrophils, the immune cells, to help sterilize and heal the injured tissue. However, since the activity of neutrophils can damage the host cells, the infiltration of neutrophils into tissue is playing a prominent part of the pathophysiology for various acute or chronic inflammations. Consequently, inhibiting neutrophil migration has been an important strategy in the development of antiinflammatory drug.

Garlic and its preparations have long been recognized to possess antiinflammatory properties $[1,2]$. Extensive in vitro studies have established that garlic oil (GO), one of the garlic extracts, is inhibitory for neutrophil transmigration through the endothelial cell monolayer [3] and for microtubule polymerization in mouse fibroblast cells [4]. It is, thus, of interest to investigate the effects of GO on the locomotion of neutrophil-like cells. In this study, we have designed and fabricated a novel chemotactic gradient Labchip to quantitatively characterize the effects of GO treatment on the locomotion of neutrophil-like cells differentiated from HL-60 cell line. In particular, the locomotion of neutrophillike cells is characterized by migration direction, average migration speed, chemotactic index (CI), and motility index (MI).

For comparison, the neutrophil-like cells with and without GO pretreatment were used as the test and the control groups, respectively. Since allicin, the major sulfurcontaining component of garlic oil, has been reported to cause microtubule disassembly in fibroblasts but has no effect on actin cytoskeleton activity [4], we further used nocodazole-treated cells and cytochalasin-B (CB)-treated cells as the positive and the negative control groups, respectively. Such arrangement is feasible because nocodazole is a microtubule polymerization inhibitor while $\mathrm{CB}$ disrupts the actin cytoskeleton of cells. Both treatments are expected to decrease the migration speed of cells. 
As to the chemotactic gradient Labchip, based on the concept of enhancing molecular diffusion by gradient generation, the current gradient generator is designed to achieve a rapidly generated, steadily maintained, and dynamically controlled chemotactic environment. In our design, the gradient slope of chemotactic concentration is controlled by changing the flow rate of the working fluid and by controlling the solution inlet in the gradient generator. This has allowed us to carry out comprehensive experimental studies on the chemotactic responsiveness of neutrophil-like cells with and without GO treatment under the conditions of varying flow rates and concentration gradients of the chemoattractant (IL-8) solution.

\section{Materials and Methods}

2.1. Cell Culture. Neutrophil-like cells were differentiated from HL-60 cell line. The HL-60 cell line was purchased from FIRDI (Taiwan, BCRC number: 60273) and maintained in RPMI-1640 medium (90\% RPMI 1640, 10\% FBS, 1\% penicillin, $1 \%$ HEPES, $1 \%$ pyruvate, $1.5 \mathrm{~g} / \mathrm{L} \mathrm{NaHCO}_{3}$, and $4.5 \mathrm{~g} / \mathrm{L}$ glucose, $\mathrm{pH}=7.4$ ) with $5 \% \mathrm{CO}_{2}$ at $37^{\circ} \mathrm{C}$. When in use, the HL-60 cell line was incubated at a concentration of $5 \times 10^{5}$ cells $/ \mathrm{mL}$ with $1.3 \%$ DMSO for 7 days. This process would differentiate the HL-60 cell line into neutrophil-like cells for use.

\subsection{Chemicals}

2.2.1. Garlic Oil. Garlic oil is an essential oil, which was extracted from garlic cloves. A clove of garlic of $1.5 \mathrm{~kg}$ was blended with $3 \mathrm{~L}$ of distilled water by using a Waring blender. The volatile components of the mixture were extracted by boiling with distilled water for 4 hours. The garlic essential oil (composed of some volatile molecules of garlic) that was carried with water vapor was then cooled down in a glass condenser. Subsequently, the condensed extract was collected. The water in the extract was then removed with anhydrous $\mathrm{Na}_{2} \mathrm{SO}_{4}$ and filtered through nitrocellulose acetate membranes [5]. Usually, an average of $3.75 \mathrm{~g}$ of GO can be extracted from $1.5 \mathrm{~kg}$ of garlic cloves. The constituents of the obtained GO were analyzed by a gas chromatography-mass spectrometry system (G1800, Hewlett Packard, Palo Alto, CA). The major organosulfur compounds of GO are diallyl sulfide, diallyl disulfide, diallyl trisulfide, and allyl methyl trisulfide. Their levels are 5, 39, 34 , and $10 \mathrm{~g} / 100 \mathrm{~g}$, respectively.

2.2.2. Reagents. The cytochalasin B (CB) powder was purchased from Sigma. The powder was dissolved in dimethylsulfoxide (DMSO) and diluted in DMSO to yield stock solutions of 10 times the working concentration. When in use, the stock CB solution was diluted and applied at a concentration of $10 \mu \mathrm{M}$ [6]. The nocodazole (methyl[5-(2-thienylcarbonyl)-1H-benzimidazol-2-yl]carbamate) powder was also purchased from Sigma. The powder was dissolved in DMSO and diluted to final concentration of $0.5 \mu \mathrm{M}$ and $2 \mu \mathrm{M}$ [4]. The chemokine IL- 8 was purchased from Sigma, as well. The solution was prepared in RPMI 1640 medium and diluted to the concentrations of $6.25 \mathrm{nM}$ and $12.5 \mathrm{nM}$.

Finally, the CB-treated, the nocodazole-treated, and the GO-treated neutrophil-like cells were separately prepared by culturing the neutrophil-like cells in a $10 \mu \mathrm{M}$ CB solution, $0.5 \mu \mathrm{M}$ and $2 \mu \mathrm{M}$ nocodazole solutions, and in different GO solutions varying from $1 \mu \mathrm{g} / \mathrm{mL} \sim 10 \mu \mathrm{g} / \mathrm{mL}$ at $37^{\circ} \mathrm{C}$ for 60 minutes, respectively. On the other hand, the normal neutrophil-like cells were directly used without any further treatment. Before injecting the treatment cells into device, we washed the cells with RPMI-1640 medium.

2.3. Experimental Setup and Cell Migration Analysis. Due to the dominating laminar flow on the microscale, the mass transport between adjacent flow streams usually relies mainly on molecular diffusion, which is usually an inherently slow process. Thus, it needs a very long flow distance and time to achieve desired concentration gradient distribution by relying purely on the mechanism of molecular diffusion. In our gradient generator design, we highlighted the concept that increasing the contact surface between two fluids and decreasing the diffusion path between them improve molecular diffusion. The new chessboard-shaped pillar structure was utilized to alter the laminar flow direction laterally to enhance the desired mass transport of microfluidics and approach the nearby downstream experimental zone with a desired chemotaxis gradient.

The schematic diagram and the dimension details of our chemotaxis gradient Labchip are shown in Figure 1(a). The Labchip consists of a cell migration region and a gradient generator. Cells were introduced into the microfluidic channel with continuous flow input, and randomly distributed in the cell migration region. In designing the gradient generator, the new chessboard-shaped pillar structure was utilized to alter the laminar flow direction laterally. This design has proved to enhance the molecular diffusion and has enabled us to achieve desired concentration gradient distributions. Through repeating the lateral alteration of the streams of buffer and chemoattractant, the chemotaxis gradient generator could have a desired continuous slope of concentration gradient in a short flow distance. Besides, it is evident that with slower inlet velocity, more chemoattractant is transported laterally. In our chemotaxis gradient Labchip, up to three different solutions could be pumped in with different flow rates from individual inlets. Thus, we can obtain dynamic gradients by varying the flow rate of each input stream. Furthermore, we can also change the gradient from top-bottom to bottom-top by controlling the solution inlets.

Gradients were characterized by using greenish fluorescence dye as shown in Figures 1(b)-1(e). We injected a $5 \mu \mathrm{M}$ FITC-Dextran $(\mathrm{MW}=10 \mathrm{kDa})$ solution from one inlet and injected DI water from the other inlet. This is feasible because the molecular weight of FITC-Dextran $(\mathrm{MW}=10 \mathrm{kDa}$ at $5 \mu \mathrm{M}$ solution) is close to the molecular weight of chemokine IL-8 (MW $=8 \mathrm{kDa}$ at $6.25 \mathrm{nM}$ solution). Figure 1(b) shows the superimposition of a green background and a schematic diagram. The background was an actual image of a green gradient made with the greenish fluorescence dye. And 


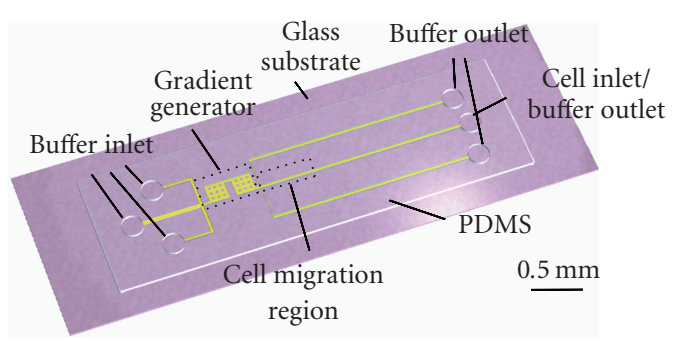

(a)

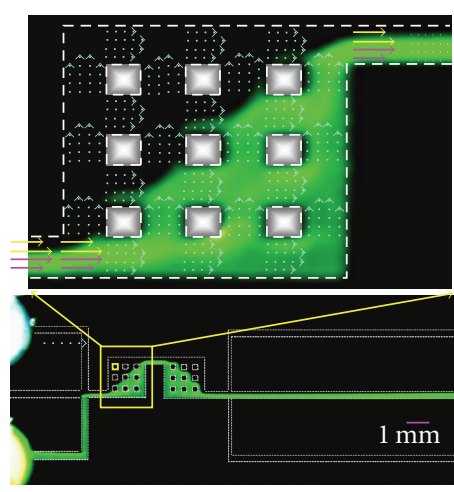

$\longrightarrow$ Solution A

$\longrightarrow$ Solution B

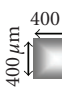

Follow direction

Square pillar

(b)

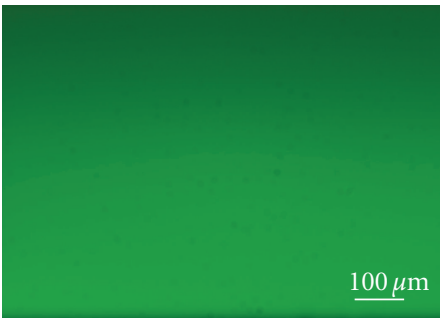

$0.5 \mu \mathrm{L} / \mathrm{min}$

(c)

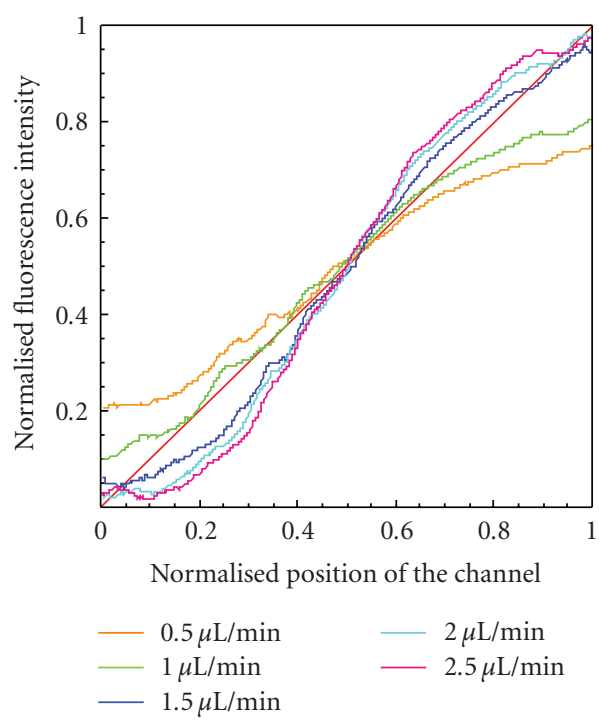

(f)

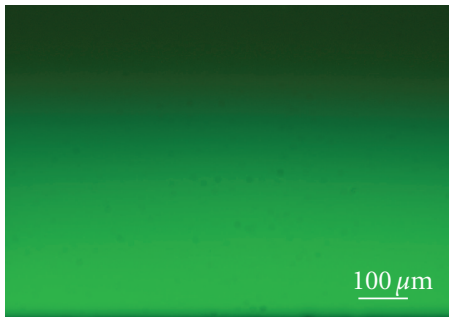

$1.5 \mu \mathrm{L} / \mathrm{min}$

(d)

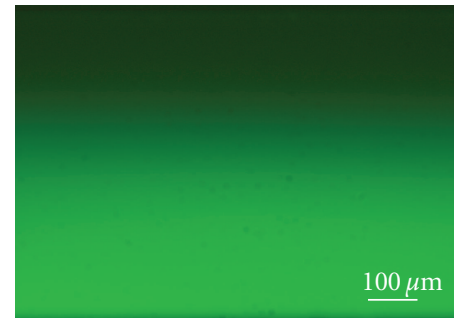

$2.5 \mu \mathrm{L} / \mathrm{min}$

(e)

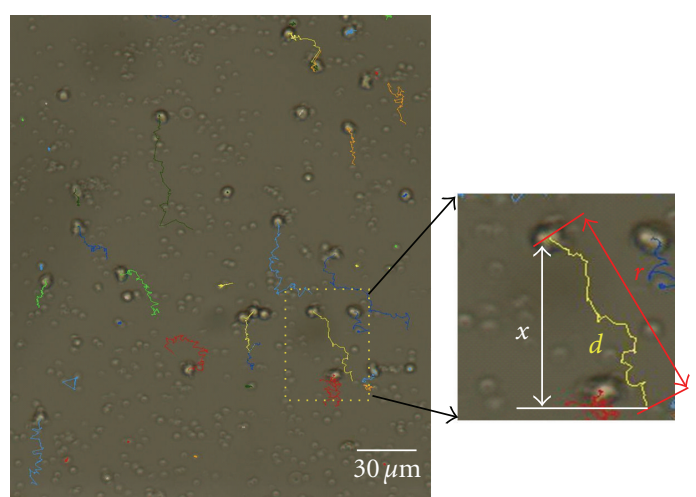

(g)

FIgURE 1: (a) The illustration of chemotactic gradient Labchip. The downstream middle outlet is used to introduce experimental cells and serves as the cell inlet at the beginning of neutrophil-like cell migration experiments. The scale bar is $0.5 \mathrm{~mm}$ long. (b) The schematic diagram illustrating the working principle of chemotaxis gradient via chessboard-shaped pillar structure. The background in green demonstrates a gradient distribution over the gradient generator via florescent medium. The scale bar is $1 \mathrm{~mm}$ long. (c), (d) and (e) The intensity pictures of the fluorescence medium at the cell migration region under three different input flow rates. The left two buffer inlets were loaded with $5 \mu \mathrm{M}$ FITC-Dextran and DI water, respectively, under the flow rates of $0.5,1.5$, and $2.5 \mu \mathrm{L} / \mathrm{min}$, separately. The scale bar is $100 \mu \mathrm{m}$ long. (f) The normalized fluorescence intensity profiles across the main channel for five different flow rates: $0.5,1,1.5,2$, and $2.5 \mu \mathrm{L} / \mathrm{min}$. (g) The image of neutrophil-like cells migration in the microchannel at the cell migration region with an IL-8 gradient of $0-6.25 \mathrm{nM}$ (from top to bottom). Lines indicate trajectories of neutrophil-like cell migration induced by the chemotaxis gradient under a flow rate of $1.5 \mu \mathrm{L} / \mathrm{min}$ (flowing from left to right) in 25 minutes. The scale bar is $30 \mu \mathrm{m}$ long. 
the schematic diagram illustrates the working principle of chemotaxis gradient via the chessboard-shaped pillar structure. The white dotted lines in the simulation figure illustrate the contour lines for the flow fields of microfluidics. The interface between the two input streams was expanded along the diagonal of each chessboard-shaped pillar unit in a short distance via the characteristics of enhanced lateral mass transport.

Figures $1(\mathrm{c})-1(\mathrm{e})$ show the recorded images of fluorescence intensity for the flow-rate conditions under 0.5 , 1.5 , and $2.5 \mu \mathrm{L} / \mathrm{min}$, respectively, at the cell migration region in our chip. Every fluorescence intensity profile was normalized to its maximum fluorescence intensity to give the normalized distribution of gradient concentration, as shown in Figure 1(f). It can be seen that the gradient slope of concentration increases when the flow rate increases.

Images of the cells in the experimental zone of neutrophil migration and chemotactic responsiveness were recorded by a microscope system with a digital CCD-camera (Mintron, MTV $62 \mathrm{~V} 1 \mathrm{~N}$, Taiwan) connected to a laptop computer and video player (Archos 404, France). A microsyringe pump (Micro 4, WPI, Florida, U.S.A) was used to pump and regulate the flow streams of fluidic sample. Fibronectin $(1 \mu \mathrm{g} / \mathrm{mL}$ solution) was injected through the cell inlet and incubated for 45 minutes at $37^{\circ} \mathrm{C}$. Neutrophil-like cells at a concentration of $5 \times 10^{6}$ cells $/ \mathrm{mL}$ were loaded uniformly across a $400 \mu \mathrm{m}$ wide main microchannel (cell migration region) and allowed to adhere within our gradient Labchip for up to 20 minutes. Adherent neutrophil-like cells within the Labchip were then exposed to three variant IL-8 solution concentration gradients: $0-6.25 \mathrm{nM}$ (slope $=$ $0.156 \mathrm{nM} / 10 \mu \mathrm{m}), 6.25-12.5 \mathrm{nM}$ (slope $=0.156 \mathrm{nM} / 10 \mu \mathrm{m})$, and $0-12.5 \mathrm{nM}$ (slope $=0.312 \mathrm{nM} / 10 \mu \mathrm{m})$ with four different flow rates, which varied from $1 \mu \mathrm{L} / \mathrm{min}-2.5 \mu \mathrm{L} / \mathrm{min}(1,1.5$, 2 , and $2.5 \mu \mathrm{L} / \mathrm{min}$ ). For our chip design, $1 \mu \mathrm{L} / \mathrm{min}$ volume flow rate was equivalent to about $330 \mu \mathrm{m} / \mathrm{sec}$ flow rate in the region of cell migration experiments. Cell movement and gradient verification were constantly monitored at a set point along the main microchannel for 45 minutes. Figure $1(\mathrm{~g})$ shows the image of neutrophil-like cells migration in the microchannel having a linear 0-6.25 nM IL-8 gradient (from the top to the bottom) under the flow rate of $1.5 \mu \mathrm{L} / \mathrm{min}$ (flowing from the left to the right). The lines in Figure 1(g) show the migration trajectories of neutrophil-like cells.

Orientation bias of cells in chemotaxis was quantified by the chemotactic index (CI) [7], which is defined as the displacement along the direction of the gradient, $x$, over the total migration distance, $d(\mathrm{CI}=x / d)$. The motility index (MI) was defined as the ratio of the displacement from starting position, $r$, and the maximum displacement, $r_{\max }$, to quantify the random motility of cells $\left(\mathrm{MI}=r / r_{\max }\right)$ [8]. Here $r_{\max }$ was the product of the average migration speed of cells and time. Besides, the effective chemotaxis was quantified by the effective chemotactic index (ECI), which is a product of $\mathrm{CI}$ and $\mathrm{MI}(\mathrm{ECI}=\mathrm{CI} \times \mathrm{MI})$.

2.4. Statistical Analysis. The data are expressed as mean \pm S.D. and were analyzed by one-way analysis of variance. Student's $t$-test was used to detect differences in means between the control group and the CB-, nocodazole-, or GOtreated group. Tukey's test was used to detect the difference among the means of the GO-treated groups. $P$ values $<.05$ were considered significant. All statistical analyses were performed with commercially available software (SAS 9.1 for Windows; SAS Institute Inc. Cary, NC, USA).

\section{Results and Discussions}

3.1. Migration of Neutrophil-Like Cells in Different Range of IL-8 Gradients. To investigate the cell migration responding to different IL-8 concentration gradients, we exposed the neutrophil-like cells to a fixed flow rate of $1.5 \mu \mathrm{L} / \mathrm{min}$ and observed the migration of neutrophil-like cells in three variant IL-8 solution concentration gradients: 0$6.25 \mathrm{nM}$ (slope $=0.156 \mathrm{nM} / 10 \mu \mathrm{m}), 6.25-12.5 \mathrm{nM}$ (slope $=$ $0.156 \mathrm{nM} / 10 \mu \mathrm{m})$, and $0-12.5 \mathrm{nM}$ (slope $=0.312 \mathrm{nM} / 10 \mu \mathrm{m})$. Figures 2(a)-2(c) show the average values of CI, MI, and ECI obtained with the abovementioned conditions for the neutrophil-like cells with and without GO treatment, respectively. In Figure 2(a), the average values of CI of the neutrophil-like cells without GO-treated are larger then that of the neutrophil-like cells with GO-treated $(P<.05)$. Under three difference IL- 8 concentration gradients, the average values of CI of cells in the control group and in GOtreated group had no significant effect. In Figure 2(b), the average values of MI among the control group and GOtreated group had no significant effect except under the 0 $12.5 \mathrm{nM}$ IL-8 concentration gradient $(P<.05)$. However, the average values of CI, MI, and ECI of neutrophil-like cells migration under the IL- 8 gradient of $0-6.25 \mathrm{nM}$ are slightly larger than those obtained with higher concentration gradients, indicating the nearly indifferent influence of IL-8 concentration on the migration activity of the neutrophillike cells. Nevertheless, by comparing the results displayed in Figures 2(a)-2(c), it does indicate the marked effect of GO treatment in suppressing the locomotion of neutrophil-like cells. The ECI value is evidently reduced by more than $50 \%$ in the whole range of IL-8 concentration gradients studied here.

3.2. Migration of Neutrophil-Like Cells under Different Continuous Flow Rates. To examine the effect of flow rate on the migration of neutrophil-like cells, the cells were exposed to different continuous flow rates of IL-8 solution. Four different flow rates, increased from $1 \mu \mathrm{L} / \mathrm{min}$ with a $0.5 \mu \mathrm{L} / \mathrm{min}$ incremental step, were used to generate $0-6.25 \mathrm{nM}$ IL-8 gradient across a $400 \mu \mathrm{m}$ wide main microchannel (Neutrophil-like cell migration region). The slopes of the IL-8 solution concentration gradients for the flow rates of $1 \mu \mathrm{L} / \mathrm{min}, 1.5 \mu \mathrm{L} / \mathrm{min}, 2 \mu \mathrm{L} / \mathrm{min}$, and $2.5 \mu \mathrm{L} / \mathrm{min}$ are $0.237,0.172,0.130$, and $0.106 \mathrm{nM} / 10 \mu \mathrm{m}$, respectively. The slope decreases with increasing flow rate. Figures 3(a)3 (c) shows the dependences of the average values of CI, MI, and ECI for the migration of the neutrophil-like cells under 0-6.25 nM IL-8 concentration gradient on flow rates as well as on GO treatment. In Figure 3(a), the average values of CI of the neutrophil-like cells without GO treatment are larger then that of the neutrophil-like cells with GO treatment 


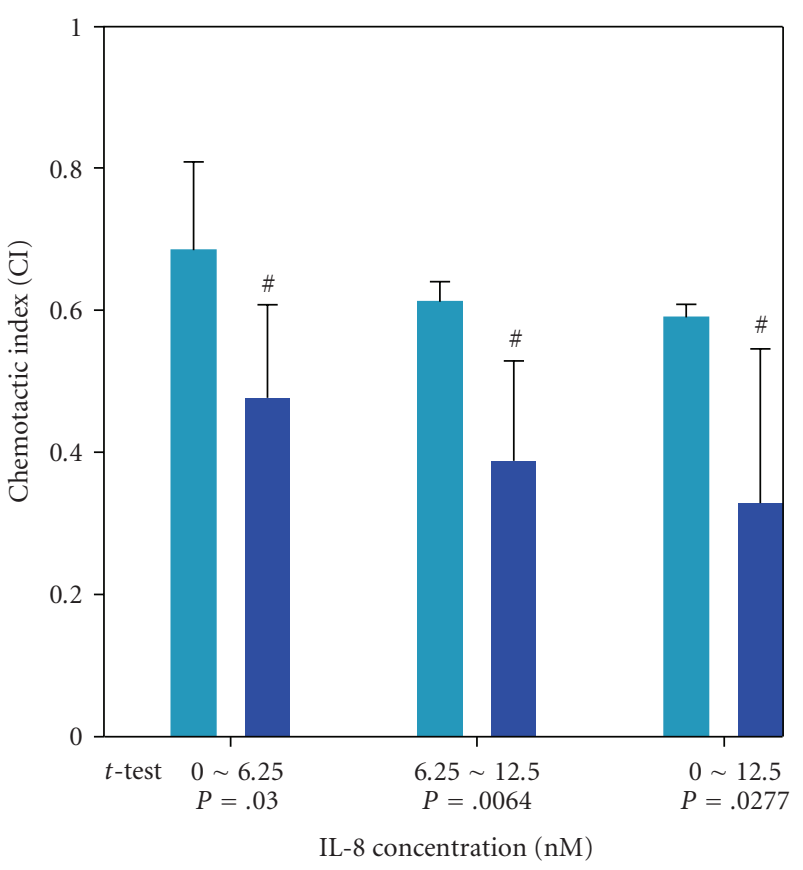

ANOVA

Concentration $P=.1261$

GO $P=.325$

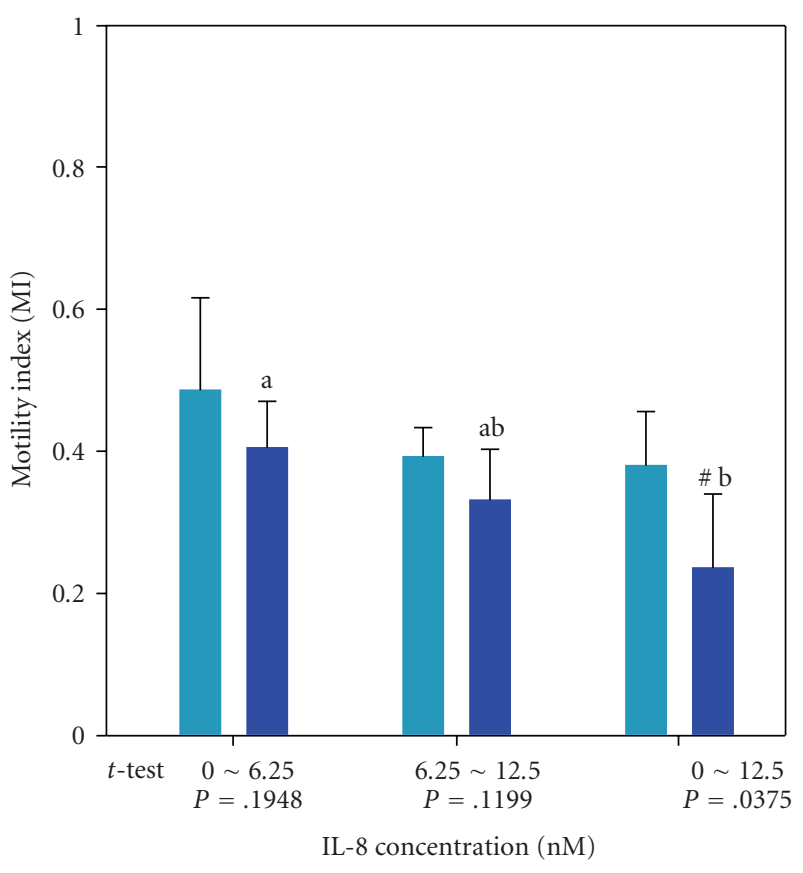

ANOVA

Concentration $P=.1603$

GO $P=.0117$

(a)

(b)

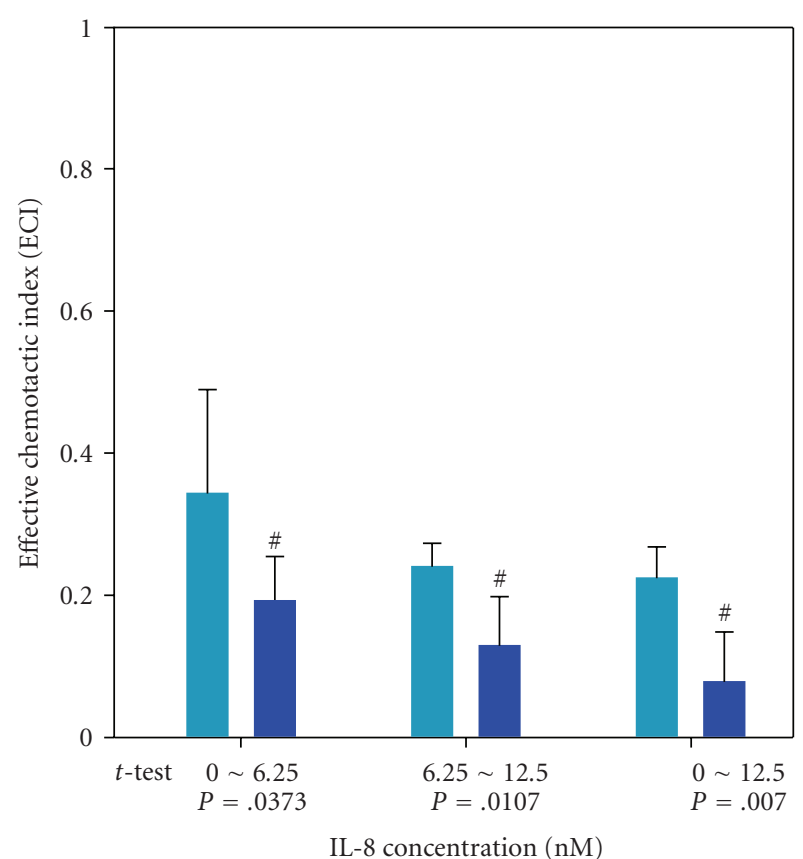

ANOVA

Concentration $P=.1001$

GO $P=.0624$

(c)

FIGURE 2: The average values of (a) CI, (b) MI, and (c) ECI of a test and a control groups of neutrophil-like cell migration under a fixed flow rate of $1.5 \mu \mathrm{L} / \mathrm{min}$ with varying IL- 8 chemoattractant gradients across a 400 - $\mu \mathrm{m}$-wide main microchannel (cell migration region). The test group of the cells was treated with $3 \mu \mathrm{g} / \mathrm{mL}$ concentration of GO while the control group was not GO-treated for comparison. Data are given in the form of mean \pm S.D. $(n=6)$. \#: Significant difference between the test and the control groups at the same range of IL- 8 gradient $(P<.05)$. a, b mean that the test groups not sharing the same superscript letter are significantly different $(P<.05)$. 


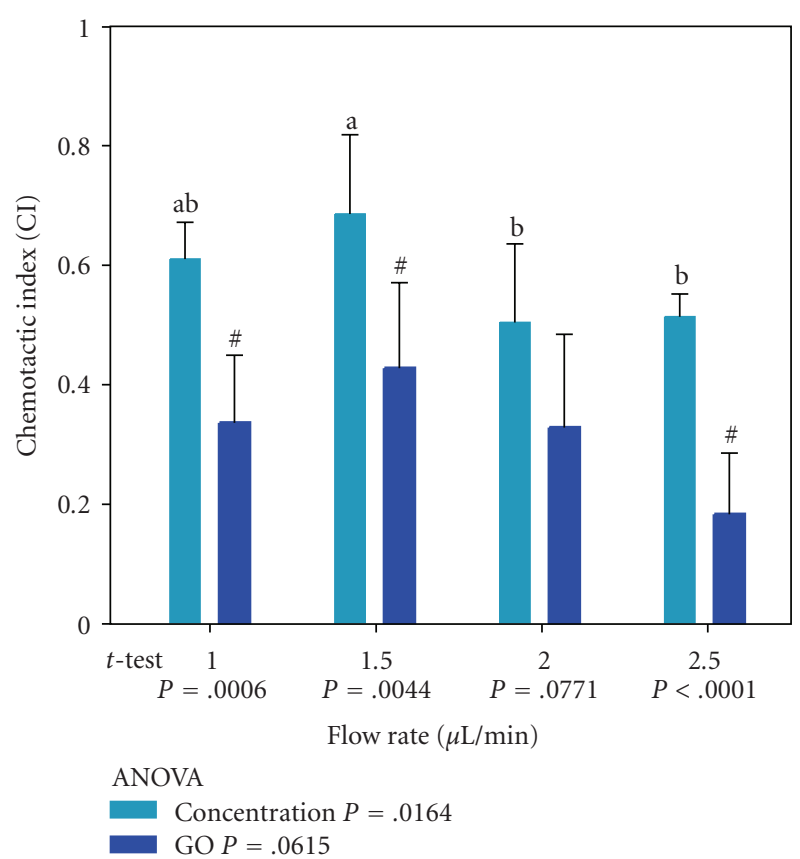

(a)

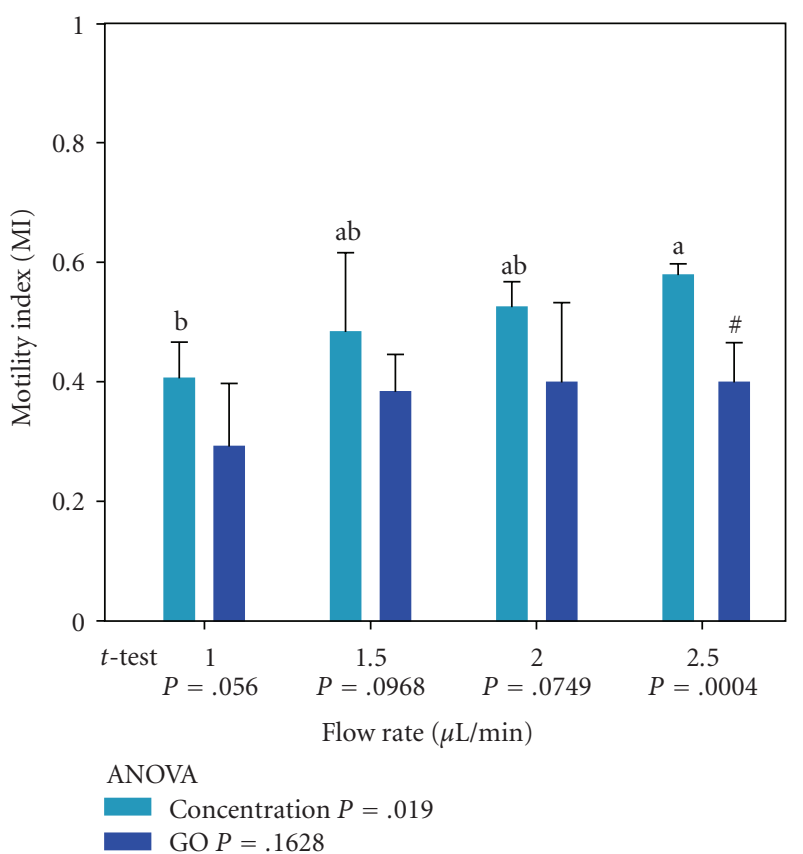

(b)

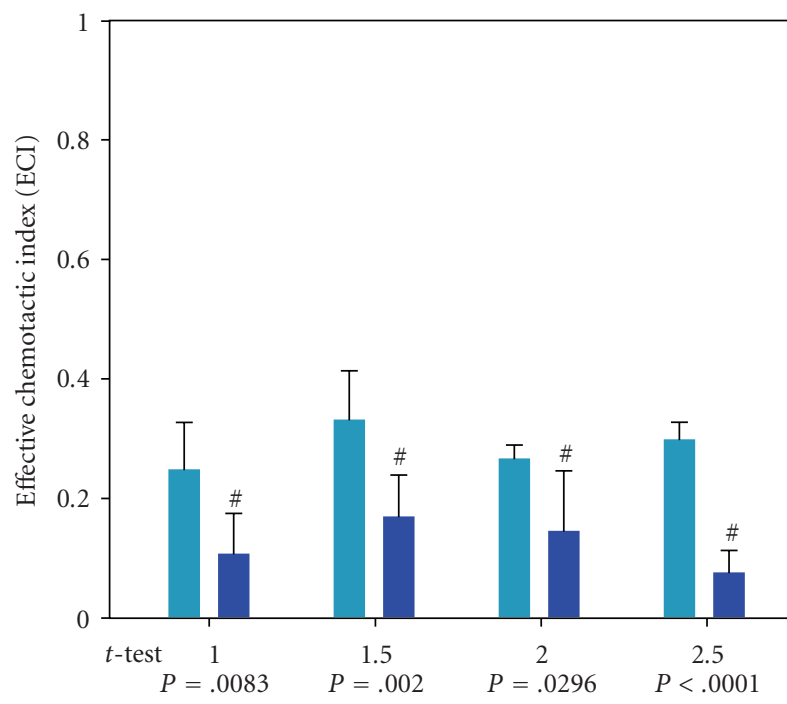

Flow rate $(\mu \mathrm{L} / \mathrm{min})$

ANOVA

Concentration $P=.1401$

GO $P=.2161$

(c)

FIGURE 3: The average values of (a) CI, (b) MI, and (c) ECI of test and control groups of neutrophil-like cell migration in the IL-8 chemoattractant gradients of $0-6.25 \mathrm{nM}$ under different flow rates across a $400-\mu \mathrm{m}$-wide main microchannel (cell migration region). The test group of the cells was treated with $3 \mu \mathrm{g} / \mathrm{mL}$ concentration of GO while the control group was not GO-treated for comparison. Data are given in the form of mean \pm S.D. $(n=6)$. \#: Significant difference between the test and the control groups at the same range of IL-8 gradient $(P<.05)$. a, b mean that the test groups not sharing the same superscript letter are significantly different $(P<.05)$.

$(P<.05)$. The average values of $\mathrm{CI}$ of the cells migration under four difference flow rates had no significant effect. In Figure 2(b), the average values of MI among the control group and the GO-treated group had no significant effect. The experimental results clearly indicate that, although the average CI value appears to reach maximum value at the flow rate of $1.5 \mu \mathrm{L} / \mathrm{min}$, the average MI value does display a trend of increasing with the flow rate regardless of whether or not cells were treated with GO. The flow rate dependence of motility is due primarily to the shear force which might 


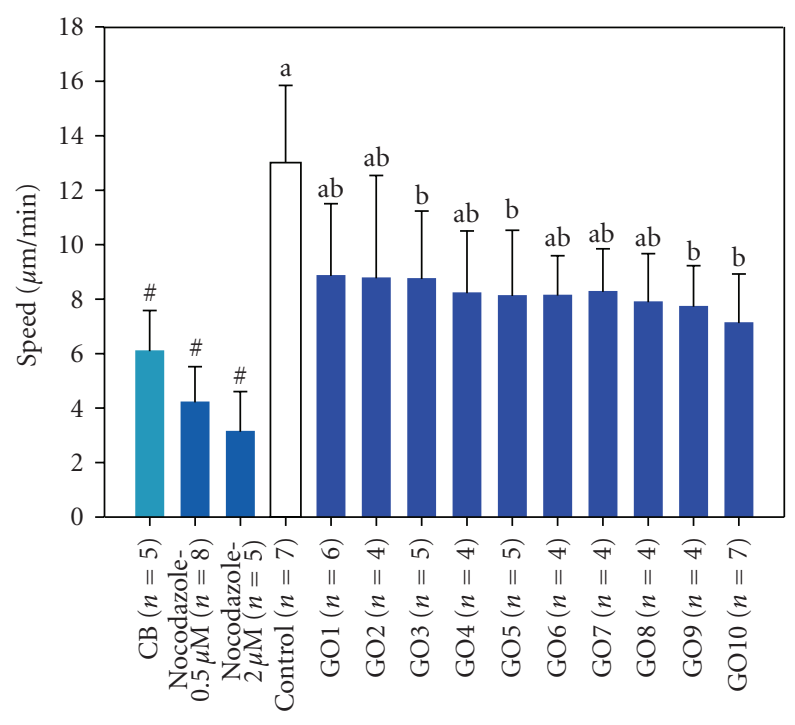

Group

(a)

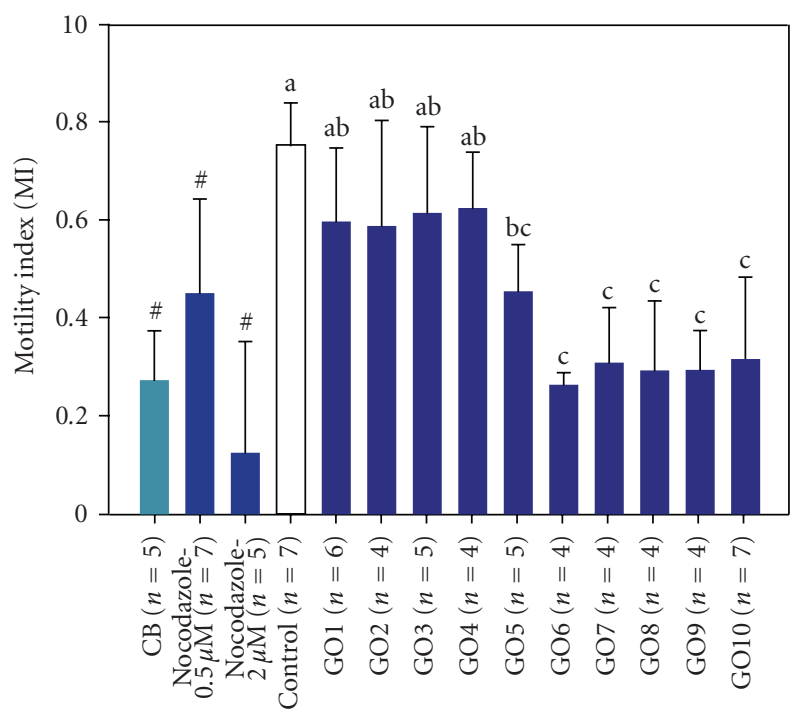

Group

(c)

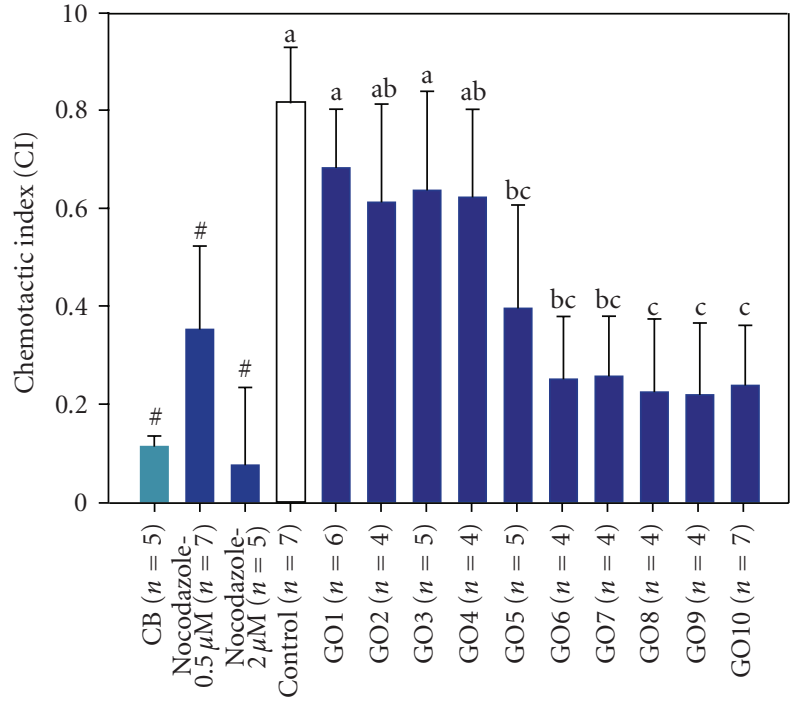

Group

(b)

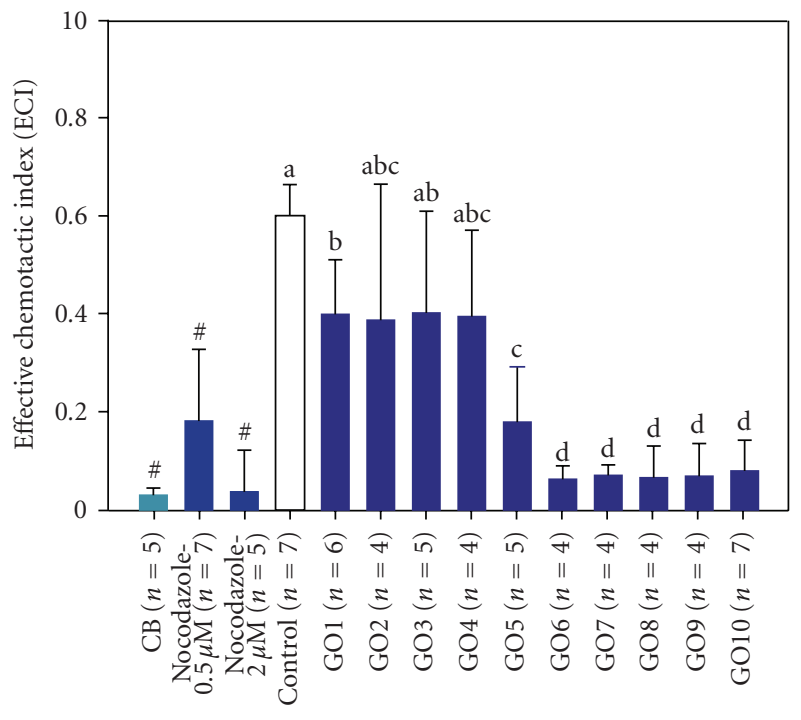

Group

(d)

FIgURE 4: The average values of (a) migration speed, (b) CI, (c) MI, and (d) ECI of fourteen groups of neutrophil-like cell migration under a fixed flow rate of $1.5 \mu \mathrm{L} / \mathrm{min}$ with an IL-8 chemoattractant gradient of $0-6.25 \mathrm{nM}$. The first four groups in each figure were the control groups for comparison. Namely, a group of CB-treated cells served as the negative control group. Two groups of nocodazole-treated cells with two different concentrations of nocodazole served as the positive control groups. And a group of cells without any treatment served as a control group. On the other hand, the following ten groups in each figure were the test groups. Each test group of cells was GO-treated with a variant GO solution concentration, ranging from $1 \mu \mathrm{g} / \mathrm{mL}$ to $10 \mu \mathrm{g} / \mathrm{mL}$. Data are given in the form of mean \pm S.D. \#: Significant difference between the control group and the CB- or nocodazole-treated group $(P<.05)$. a, b, $\mathrm{c}, \mathrm{d}$ mean that the test groups not sharing the same superscript letter are significantly different $(P<.05)$.

have driven the neutrophil-like cells downstream from the main microchannel along the direction of flow. A similar effect, however, may also deteriorate the cell migration. As a result, there appears to be an optimal operation condition for the present microfluidic gradient generation chip. In this case, $1.5 \mu \mathrm{L} / \mathrm{min}$ flow rate seems to give the best operation condition. It is emphasized that in every condition the effect of GO treatment on suppressing the cell migration is evidently demonstrated.

\subsection{The Effect of Garlic Oil Concentration on the Migration of} Neutrophil-Like Cells. To further elucidate the effect of GO on the migration of the neutrophil-like cells, we examined the migration of neutrophil-like cells treated with different 
GO concentrations in a $0-6.25 \mathrm{nM}$ IL- 8 gradient at a flow rate of $1.5 \mu \mathrm{L} / \mathrm{min}$. Figure 4 (a) shows the migration speeds of the neutrophil-like cells treated with $\mathrm{CB}$, nocodazole, and varied GO concentrations under the same measurement conditions as described above. The average migration speed of normal neutrophil-like cells obtained in the current study is about $13 \mu \mathrm{m} / \mathrm{min}$. Compared to the previous nonfluidic experiments $[9,10]$ performed in a three-dimensional collagen matrix, in which the maximum migration speed of $15 \sim 20 \mu \mathrm{m} / \mathrm{min}$ for human neutrophil cells was reported, the present results evidently demonstrate that our microfluidic chemotaxis gradient Labchip is indeed viable for studying the cell migration and chemotactic responses. Figure 4(a) also shows that the average migration speeds of neutrophillike cells are reduced from $13 \mu \mathrm{m} / \mathrm{min}$ for the untreated cells to $6 \mu \mathrm{m} / \mathrm{min}, 4 \mu \mathrm{m} / \mathrm{min}$, and $8 \mu \mathrm{m} / \mathrm{min}$ after being treated with $\mathrm{CB}$, nocodazole, and GO, respectively. The results unambiguously demonstrate that $\mathrm{CB}$ as well as nocodazole treatments are more effective than GO treatment in inhibiting the migration of neutrophil-like cells. It is also interesting to note that the migration inhibition appears to be rather insensitive to the GO concentration, which certainly requires further investigations. In any case, the present results evidently confirm the fundamental mechanisms practiced in developing the antiinflammatory drugs.

In addition to reducing the migration speed of the neutrophil-like cells, the $\mathrm{CB}$, nocodazole, and GO treatments also display very similar effects in reducing the average $\mathrm{CI}$, MI, and ECI values for the neutrophil-like cells. As shown in Figure 4(b), the cells treated with CB or nocodazole exhibit an immediate reduction in the average values of CI, MI, and ECI. (Note that $P<.05$ for the difference between the two control groups: one with CB treatment and the other without any treatment. Similarly, $P<.05$ for the difference between the nocodazole-treated group and the group without any treatment.) On the other hand, for those treated with GO, significant effects are observed only when the concentration of GO exceeds $5 \mu \mathrm{g} / \mathrm{mL}$. (Note that $P<.05$ for the difference between the test groups with GO exceeding $5 \mu \mathrm{g} / \mathrm{mL}$ and the control group without any treatment.)

According to the generally conceived pathophysiology of various acute or chronic inflammations described previously, the activities for neutrophils to transmigrate through the walls of blood vessels and to infiltrate into the damaged tissues are primarily relying on the cytoskeleton rearrangement of these cells. This is in excellent agreement with what has been observed here. Our results are consistent with the findings by Prager-Khoutorsky et al. [4] who reported that allicin, the major sulfur-containing composition of garlic oil, directly affects the activity of cytoskeleton of fibroblast cells by reaction with tubulin $\mathrm{SH}$ groups. Consequently, we speculated that the inhibitory effect of GO on the migration of neutrophil-like cells is at least partly due to an effect on cytoskeleton activity. Nevertheless, the effect of GO on other mechanisms associated with neutrophil migration may not be excluded. In addition, the fact that GO treatment displays similar effects in retarding the migration speed of neutrophillike cells further implies that similar mechanisms are prevailing when enough GO is introduced. In other words, GO might also play a prominent role in interrupting the assembly and disassembly processes of the cytoskeleton inside the cell as well, even though direct evidence and detailed mechanisms are still lacking at present. It is anticipated that, with the capabilities of obtaining quantitative cell migration dynamical parameters with the novel Labchip demonstrated in this study, the development of antiinflammatory drugs can be realized in a much more efficient manner.

\section{Conclusions}

In summary, we have designed and fabricated a chemotactic gradient Labchip which enables us to conduct quantitative investigations on the effects of garlic oil treatment on the migration properties of the neutrophil-like cells. The preliminary results unambiguously demonstrate not only the capabilities of the present gradient Labchip in generating desired concentrations gradient and flow rate in a controlled manner but also in obtaining meaningful quantitative results consistent with those obtained from nonfluidic three-dimensional collagen matrix. The experimental data evidently show that the average migration speed of cells is reduced after being treated with GO. The results suggest that garlic oil is a potential inhibitor for neutrophil-like cell migration which, in turn, could result in antiinflammatory activities through the inhibition of the assembly and disassembly of the cytoskeleton inside the cell.

\section{Acknowledgments}

This work was financially supported by the Nanotechnology Research Program of the University System and the National Science Council of Taiwan, R.O.C. under Grants: 96-2120M-009-003 and 97-2120-M-007-011. The authors thank Dr. Hwei-Ling Peng, Dr. Hwan-You Chang, Dr. Ying-Jung Hung, Dr. Ke-Ming Chen, and Kou-Chuan Huang for helpful comments and discussion. The first two authors contributed equally to this work.

\section{References}

[1] K. T. Augusti, "Therapeutic values of onion (Allium cepa L.) and garlic (Allium sativum L.)," Indian Journal of Experimental Biology, vol. 34, no. 7, pp. 634-640, 1996.

[2] K. C. Agarwal, "Therapeutic actions of garlic constituents," Medicinal Research Reviews, vol. 16, no. 1, pp. 111-124, 1996.

[3] R. Hofbauer, M. Frass, B. Gmeiner, A. D. Kaye, and E. A. Frost, "Effects of garlic extract (Allium sativum) on neutrophil-like cell migration at the cellular level," Heart Disease, vol. 3, pp. 14-17, 2001.

[4] M. Prager-Khoutorsky, I. Goncharov, A. Rabinkov, D. Mirelman, B. Geiger, and A. D. Bershadsky, "Allicin inhibits cell polarization, migration and division via its direct effect on microtubules," Cell Motility and the Cytoskeleton, vol. 64, no. 5, pp. 321-337, 2007.

[5] L. Y. Sheen, S. Y. Lin, and S. J. Tsai, "Odor assessments for volatile compounds of garlic and ginger essential oils by sniffing method of gas chromatography," Journal of the Chinese Agricultural Chemical Society, vol. 30, pp. 14-24, 1992. 
[6] C. Rotsch and M. Radmacher, "Drug-induced changes of cytoskeletal structure and mechanics in fibroblasts: an atomic force microscope study," Biophysical Journal, vol. 78, no. 1, pp. 520-535, 2000.

[7] P. V. Moghe, R. D. Nelson, and R. T. Tranquillo, "Cytokinestimulated chemotaxis of human neutrophils in a 3-D conjoined fibrin gel assay," Journal of Immunological Methods, vol. 180, no. 2, pp. 193-211, 1995.

[8] F. Lin, C. M. C. Nguyen, S. J. Wang, W. Saadi, S. P. Gross, and N. L. Jeon, "Effective neutrophil chemotaxis is strongly influenced by mean IL-8 concentration," Biochemical and Biophysical Research Communications, vol. 319, no. 2, pp. 576-581, 2004.

[9] J. Werr, X. Xie, P. Hedqvist, E. Ruoslahti, and L. Lindbom, “ $\beta 1$ integrins are critically involved in neutrophil locomotion in extravascular tissue in vivo," Journal of Experimental Medicine, vol. 187, no. 12, pp. 2091-2096, 1998.

[10] F. Entschladen, M. Gunzer, C. M. Scheuffele, B. Niggemann, and K. S. Zanker, "T lymphocytes and neutrophil granulocytes differ in regulatory signaling and migratory dynamics with regard to spontaneous locomotion and chemotaxis," Cellular Immunology, vol. 199, no. 2, pp. 104-114, 2000. 

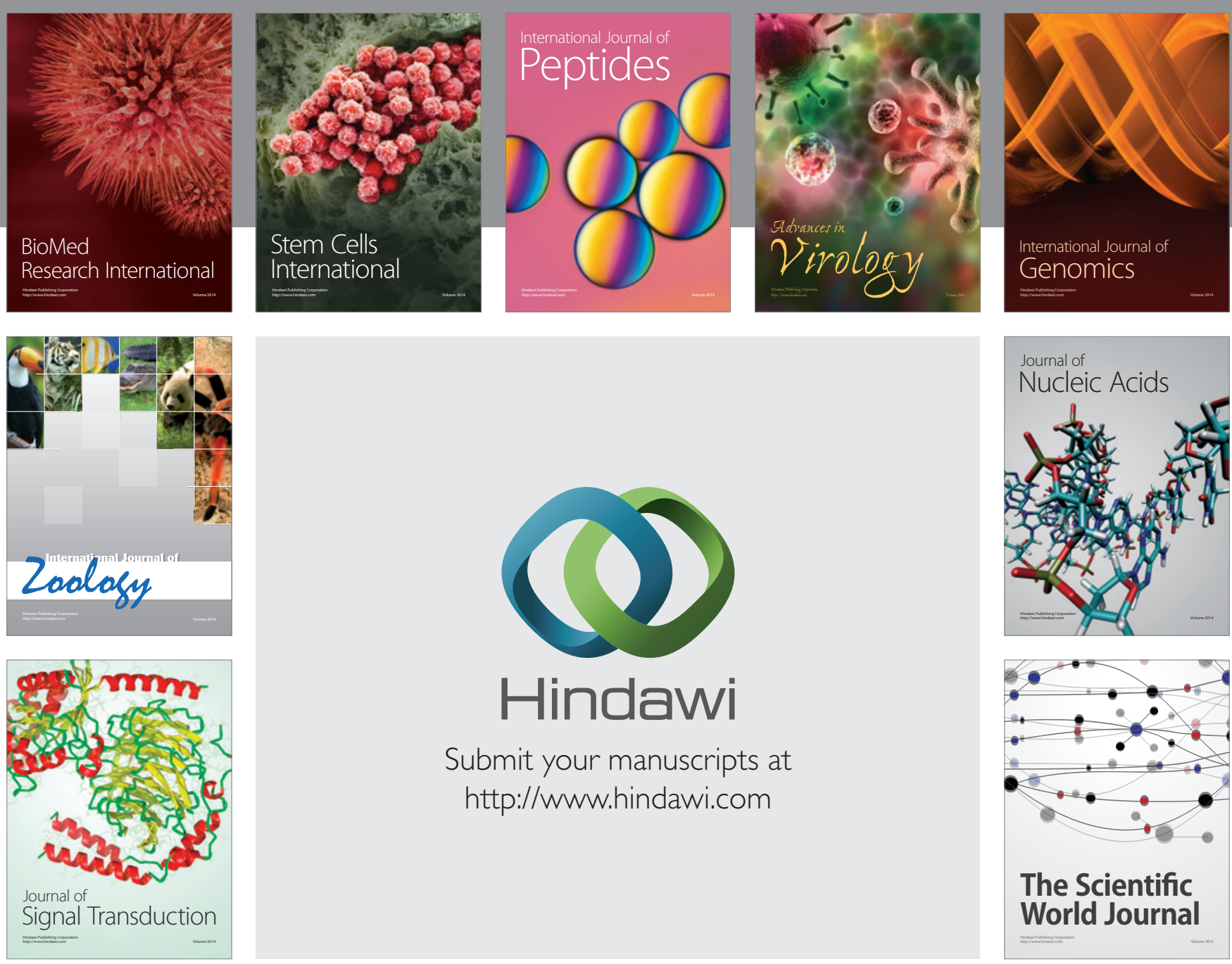

Submit your manuscripts at

http://www.hindawi.com
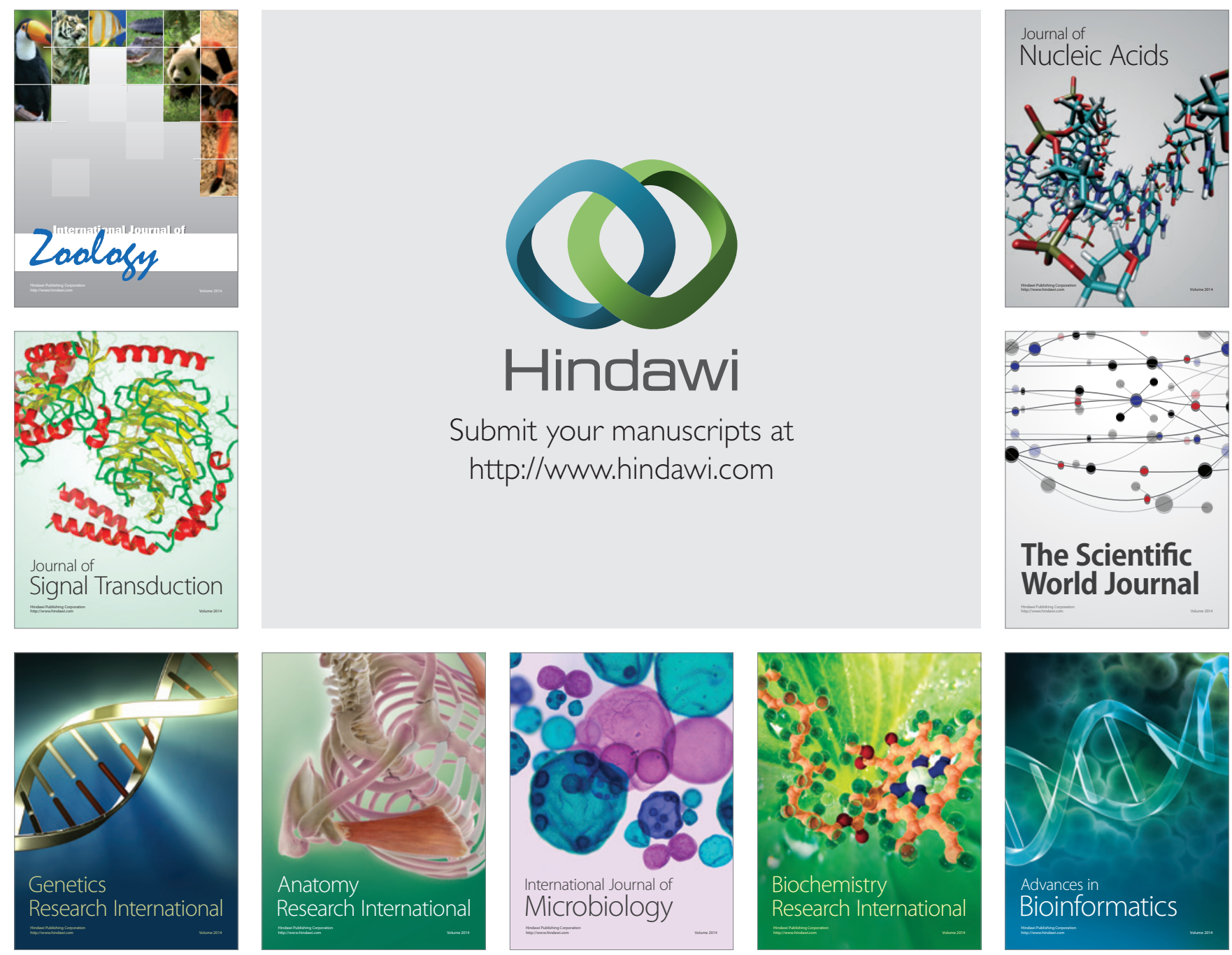

The Scientific World Journal
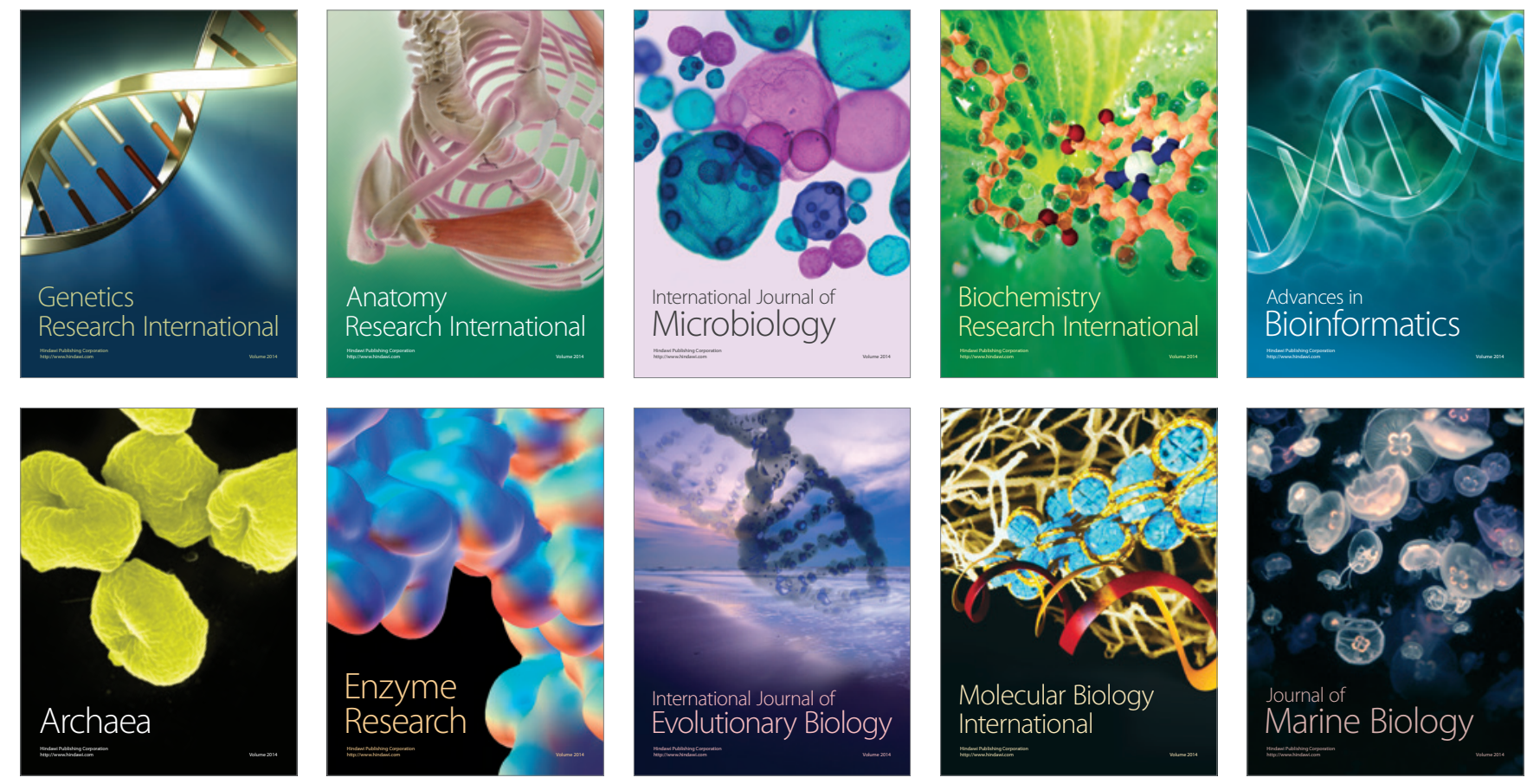